\title{
SOME OPTIMIZATION AND DECISION PROBLEMS IN PROPORTIONAL REINSURANCE
}

\author{
ANNA CASTAÑER \\ acastaner@ub.edu \\ Universitat de Barcelona, Dept. Matemàtica Econòmica, Financera i Actuarial, \\ Avenida Diagonal, 690, 08034, Barcelona, Spain \\ M. MERCÈ CLARAMUNT \\ mmclaramunt@ub.edu \\ Universitat de Barcelona, Dept. Matemàtica Econòmica, Financera i Actuarial, \\ Avenida Diagonal, 690, 08034, Barcelona, Spain \\ MAITE MÁRMOL \\ mmarmol@ub.edu \\ Universitat de Barcelona, Dept. Matemàtica Econòmica, Financera i Actuarial, \\ Avenida Diagonal, 690, 08034, Barcelona, Spain \\ Recibido $(30 / 03 / 2016)$ \\ Revisado $(20 / 08 / 2016)$ \\ Aceptado (10/10/2016)
}

RESUMEN: El reaseguro es uno de los instrumentos que un asegurador puede utilizar para mitigar el riesgo de suscripción y por lo tanto controlar su solvencia. En este artículo, nos centramos en los contratos de reaseguro proporcional y examinamos diversos problemas de optimización y decisión del asegurador respecto de la estrategia de reaseguro. Con este fin, utilizamos como instrumentos de decisión no sólo la probabilidad de ruina sino también la variable aleatoria déficit en el momento de ruina si la ruina ocurre. La función de penalización descontada se utiliza para calcular como casos particulares la probabilidad de ruina, y los momentos y la función de distribución del déficit en el momento de ruina si la ruina ocurre.

Consideramos el modelo de la teoría clásica del riesgo asumiendo un proceso de Poisson y una cuantía individual de los siniestros distribuida según una phase-type, modificado con un reaseguro proporcional con un nivel de retención que no es constante y que depende del nivel de las reservas. La función de penalización descontada se comporta diferente según si el nivel inicial de las reservas está por encima o por debajo de un determinado umbral. Se obtienen expresiones generales para esta función de penalización descontada, así como resultados teóricos interesantes y expresiones explícitas para el caso phase-type 2. Estos resultados se aplican en ejemplos numéricos de problemas de decisión basados en la probabilidad de ruina y en diferentes medidas de riesgo del déficit en el momento de ruina si la ruina ocurre (esperanza, Value at risk y Tail Value at Risk.

Palabras Clave: Optimización, Distribución phase-type, Reaseguro.

ABSTRACT: Reinsurance is one of the tools that an insurer can use to mitigate the underwriting risk and then to control its solvency. In this paper, we focus on the proportional reinsurance arrangements and we examine several optimization and decision problems of the insurer with respect to the reinsurance strategy. To this end, we use as decision tools not only the probability of ruin but also the random variable deficit at ruin if ruin occurs. The discounted penalty function is employed to calculate as particular cases the probability of ruin and the moments and the distribution function of the deficit at ruin if ruin occurs.

We consider the classical risk theory model assuming a Poisson process and an individual claim amount phase-type distributed, modified with a proportional reinsurance with a retention level that is not constant and depends on the level of the surplus. Depending on whether the initial surplus is below or above a threshold level, the discounted penalty function behaves differently. General expressions for this discounted penalty function are obtained, as well as interesting theoretical results and explicit expressions for phasetype 2 distribution. These results are applied in numerical examples of decision problems based on the probability of ruin and on different risk measures of the deficit at ruin if ruin occurs (the expectation, the Value at Risk and the Tail Value at Risk)

Keywords: Optimality, Phase-type distribution, Reinsurance. 


\section{Introduction}

The influence of reinsurance strategies on the solvency of the insurer is an important subject and has been widely analyzed in actuarial literature. A common approach is to minimize some measure of the insurer risk after reinsurance (see e.g. $[1,2,3,4,5,6,7,8]$ ). Several optimization problems have been considered using different kinds of reinsurance strategies, being the proportional, the excess of loss and the stop-loss the most well-known (see [9] and the references therein).

One of the main measures used to control solvency is ruin probability, but in this paper we use also other measures related to the deficit at ruin if ruin occurs, as its expectation or the Value at Risk $(V a R)$ or the Tail Value at Risk $(T V a R)$. We study the random variable deficit at ruin if ruin occurs in the classical risk theory model considering a proportional reinsurance arrangement, where the retention level is not constant and depends on the level of the surplus. This type of reinsurance, called threshold proportional reinsurance, has been first defined and studied in [3,4], and includes, as a particular case, the classical proportional reinsurance with constant retention level.

The main objective of this paper is to study the effect of the threshold proportional reinsurance on the probability of ruin and on the other risk measures related with the deficit at ruin. The Gerber-Shiu function $[10,11]$ is used as the mathematical tool in order to obtain general results that can be translated into explicit expressions for phase-type 2 distribution. We also perform a comparative analysis with the proportional reinsurance. Our results can assist the insurer in his reinsurance decision process concerning solvency (related optimality problems in reinsurance can be found for instance in $[12,13,14,15])$.

After this introduction, the paper is organized as follows. In Sections 2 and 3, notation, assumptions and preliminaries are included. In Section 4.1, we present some general results for the Gerber-Shiu function for the ruin probability, the ordinary moments and the distribution function of the deficit at ruin if ruin occurs, when the individual claim amount follows a general phasetype distribution. In Section 4.2, we assume a phase-type 2 distribution and analyzed the previous results. Then, the explicit expressions are obtained. An interesting result about the distribution of the deficit at ruin if ruin occurs in a model with a threshold reinsurance is demonstrated in Proposition 3. In Section 5, some optimization and decision problems of the reinsurance strategy are presented. In this analysis, the ruin probability and the deficit at ruin if ruin occurs are used as decision tools for the insurer. This section includes some numerical examples. Section 6 closes the paper giving some concluding remarks.

\section{Notation}

In the classical risk theory model, the surplus, $R(t)$, at a given time $t \in[0, \infty)$ is defined as $R(t)=u+c t-S(t)$, with $u=R(0) \geq 0$ being the insurer's initial surplus, $S(t)$ the aggregate claims and $c$ the instantaneous premium rate. $S(t)$ is modeled as a compound Poisson process

$$
S(t)=\sum_{i=1}^{N(t)} X_{i} .
$$

The claim number process $\{N(t)\}_{t \geq 0}$ is assumed to be Poisson with parameter $\lambda$. Specifically, the corresponding claim inter-arrival times, denoted by $\left\{T_{i}\right\}_{i=1}^{\infty}$ are independent and identically distributed (i.i.d.) exponentially distributed random variables with parameter $\lambda$, where $T_{1}$ denotes the time until the first claim and $T_{i}$, for $i>1$, denotes the time between the $(i-1)$ th and $i$ th claims. The random variables $\left\{X_{i}\right\}_{i=1}^{\infty}$ are the positive claim severities, which are i.i.d. random variables with common probability density function $f(x)$ and distribution function $F(x)$, and $\{N(t)\}_{t \geq 0}$ is independent of $\left\{X_{i}, i \geq 1\right\}$. We assume that the insurer's premium income is received continuously 
at rate $c$ per unit time, where $c=\lambda E[X](1+\rho)$, and $\rho>0$ is the premium loading factor.

The time of ruin is $T=\min \{t \geq 0 \mid R(t)<0\}$, with $T=\infty$ if $R(t) \geq 0$ for all $t \geq 0$. The deficit at ruin if ruin occurs is $Y=|R(T)|$ and the surplus immediately prior to ruin is $R(T-)$. The probability of ultimate ruin from initial surplus $u$ is denoted $\psi(u)$ and defined by

$$
\psi(u)=P[T<\infty \mid R(0)=u]=E\{I(T<\infty) \mid R(0)=u\},
$$

where $I(A)=1$ if $A$ occurs and $I(A)=0$ otherwise.

Gerber and Shiu [10] introduced the Gerber-Shiu discounted penalty function $\phi(u)$,

$$
\phi(u)=E\left[e^{-\delta T} w(R(T-),|R(T)|) I(T<\infty) \mid R(0)=u\right],
$$

being $\delta \geq 0$ the discounted factor, and $w(l, j), l \geq 0, j>0$, the penalty function, so that $\phi(u)$ is the expected discounted penalty payable at ruin. This function is known to satisfy a defective renewal equation $[10,11]$. This function can be used to study the traditional quantities of interest in classical ruin theory, such as ruin probability, time of ruin or deficit at ruin. Therefore, depending on the penalty function $w(l, j)$, we can obtain different interpretations for the Gerber-Shiu function:

i) For $w(l, j)=1$,

$$
\phi(u)=E\left[e^{-\delta T} I(T<\infty) \mid R(0)=u\right],
$$

i.e. the defective Laplace transform of the time of ruin being $\delta$ the parameter. In addition, if we consider $\delta=0$, the ultimate ruin probability is obtained

$$
\phi(u)=\psi(u) \text {. }
$$

ii) For $w(l, j)=j^{m}$ and $m \geq 1$,

$$
\phi(u)=E\left[e^{-\delta T} Y^{m} I(T<\infty) \mid R(0)=u\right],
$$

and dividing this Gerber-Shiu function by the probability of ruin, the ordinary discounted moments of the deficit at ruin if ruin occurs are obtained,

$$
\alpha_{m}(Y)=\frac{E\left[e^{-\delta T} Y^{m} I(T<\infty) \mid R(0)=u\right]}{\psi(u)} .
$$

If we let $\delta=0$ in (2), the ordinary moments of the deficit at ruin if ruin occurs are obtained.

iii) For $w(l, j)=I(j \leq y)$,

$$
\phi(u)=E\left[e^{-\delta T} I(Y \leq y) I(T<\infty) \mid R(0)=u\right]
$$

and dividing by the probability of ruin we obtain the distribution function of the discounted deficit at ruin if ruin occurs,

$$
F_{Y}(y)=\frac{E\left[e^{-\delta T} I(Y \leq y) I(T<\infty) \mid R(0)=u\right]}{\psi(u)} .
$$

If we let $\delta=0$, the distribution function of the deficit at ruin if ruin occurs is obtained.

In this paper we analyze the deficit at ruin in the classical risk theory model assuming a compound Poisson process for the aggregate claims and a phase-type distribution for the individual claim amount, when the insurer considers a threshold proportional reinsurance.

The threshold proportional reinsurance strategy [4] is a dynamic strategy with a retention level that is not constant and depends on the level of the surplus, $R(t)$. A retention level $k_{1}$ is applied whenever the reserves are less than a threshold $b \geq 0$, and a retention level $k_{2}$ is applied in the other case. Then, the premium income retained is $c_{1}$ and $c_{2}$, respectively. We consider that the 
retention levels give new positive security loadings for the insurer, i.e. the net profit condition is always fulfilled. Then, we can define $\rho_{1}=\rho_{R}-\frac{\rho_{R}-\rho}{k_{1}}$ and $\rho_{2}=\rho_{R}-\frac{\rho_{R}-\rho}{k_{2}}, \rho_{R}$ being the loading factor of reinsurer.

\section{Assumptions and preliminaries}

In our model with threshold proportional reinsurance strategy, the discounted penalty function (1) behaves differently, depending on whether initial surplus $u$ is below or above the level $b$. Hence, for notational convenience, we write

$$
\phi(u)=\left\{\begin{array}{lr}
\phi_{1}(u), & 0 \leq u<b, \\
\phi_{2}(u), & u \geq b .
\end{array}\right.
$$

In [3] a theorem for the integro-differential equation for the Gerber-Shiu function (1) is obtained in a Poisson model for the claim process. We include this theorem in order that the paper is selfcontained, taking into account that we will use it in the next sections.

Theorem 1 The discounted penalty function $\phi(u)$ in a Poisson process model satisfies the integrodifferential equations

$$
\phi^{\prime}(u)=\left\{\begin{array}{l}
\phi_{1}^{\prime}(u), 0<u<b \\
\phi_{2}^{\prime}(u), \quad u>b
\end{array}\right.
$$

where

$$
\begin{aligned}
\phi_{1}^{\prime}(u) & =\frac{\lambda+\delta}{c_{1}} \phi_{1}(u)-\frac{\lambda}{c_{1}} \int_{0}^{\frac{u}{k_{1}}} \phi_{1}\left(u-k_{1} x\right) d F(x)-\frac{\lambda}{c_{1}} \xi_{1}(u), \\
\phi_{2}^{\prime}(u) & =\frac{\lambda+\delta}{c_{2}} \phi_{2}(u)-\frac{\lambda}{c_{2}}\left[\int_{0}^{\frac{u-b}{k_{2}}} \phi_{2}\left(u-k_{2} x\right) d F(x)\right. \\
& \left.+\int_{\frac{u-b}{k_{2}}}^{\frac{u}{k_{2}}} \phi_{1}\left(u-k_{2} x\right) d F(x)\right]-\frac{\lambda}{c_{2}} \xi_{2}(u)
\end{aligned}
$$

and

$$
\xi_{1}(u)=\int_{\frac{u}{k_{1}}}^{\infty} w\left(u, k_{1} x-u\right) f(x) d x, \quad \xi_{2}(u)=\int_{\frac{u}{k_{2}}}^{\infty} w\left(u, k_{2} x-u\right) f(x) d x .
$$

Let $w(R(T-),|R(T)|)$ be a non-negative function of $R(T-)>0$, the surplus immediately before ruin, and $|R(T)|>0$ the surplus at ruin.

As we focus our analysis on the deficit at ruin if ruin occurs, we will consider only a specific subgroup of penalty functions

$$
W D=\left\{w(l, j)=j^{m}, w(l, j)=I(j \leq y), w(l, j)=1\right\}
$$

with $m>0$.

We assume that the individual claim amount follows a phase-type distribution $P H(v, S)$. Key results of modern theory of phase-type distributions including theoretical properties, characterization and applications can be found in $[16,17,18,19,20]$. Most of the original applications of phase-type distributions are in queuing theory, but these kind of distributions are widely used in risk theory in the last years.

In [21] many applications in this field can be found. Other important references on phase-type distributions in risk theory context include $[22,23,24,25,26,27]$. We present a brief overview of phase-type distributions and their properties. 
Phase-type distributions: We consider a continuous time Markov chain with a single absorbing state 0 and $N$ transient states. The row vector $v$ contains the probabilities $\alpha_{j}$ that the process starts in the various transient states $j=1,2, \ldots, N$. If the probability of starting the process in the absorbing state is zero, $\sum_{j=1}^{N} \alpha_{j}=1$. Then, $v e^{\top}=1$ where $e^{\top}$ is a column vector of ones with $N \times 1$ elements.

The infinitesimal generator $Q$ for the continuous time Markov chain is given by

$$
Q=\left(\begin{array}{cc}
\mathbf{0} & 0 \\
S^{0} & S
\end{array}\right)
$$

where $S$ is the matrix of transition rates among the transient states and $S^{0}$ is the column vector of absorption rates into state 0 from the transient states. Necessarily, $S^{0}=-S e^{\top}$, and $S$ is an $N \times N$ matrix whose diagonal entries are negative and whose other entries are non-negative. Under these assumptions, the distribution of time $X$ until the process reaches the absorbing state is said to be phase-type distributed and is denoted $P H(v, S)$ with distribution $F_{X}(x)=1-v \exp (S x) e^{\top}$ for $x \geq 0$, density function $f_{X}(x)=v \exp (S x) S^{0}$ for $x>0$ and ordinary moments $\alpha_{m}(X)=$ $(-1)^{m} m ! v S^{-m} e^{\top}$, being $\exp (\cdot)$ the matrix exponential.

The Laplace transform of the density function $\tilde{f}_{X}(t)=\int_{0}^{\infty} e^{-t x} f_{X}(x) d x$ is a rational function of degree $\leq N$

$$
\tilde{f}_{X}(t)=\frac{a(t)}{b(t)}
$$

with $a(t)=\sum_{i=0}^{N} a_{i} t^{i}, a_{0}=0, b(t)=\sum_{i=0}^{N} b_{i} t^{i}, b_{0}=1$ and $f_{X}(x)$ satisfies the linear differential equation

$$
\sum_{i=0}^{N} b_{i} f_{X}^{(i)}(x)=0
$$

The finite mixture of phase-type distributions is a phase-type distribution. Let $X_{i}, i=1, \ldots, k$ be distributed as $P H\left(v_{i}, S_{i}\right)$, and $Y=I_{i} X_{i}$ being $\sum_{i=1}^{k} I_{i}=1$, and $P\left(I_{i}=1\right)=p_{i}$ then $Y$ is $P H(v, S)$ with

$$
v=\left(p_{1} v_{1}, \ldots, p_{k} v_{k}\right) \quad \text { and } \quad S=\left(\begin{array}{cccc}
S_{1} & 0 & \cdots & 0 \\
0 & S_{2} & \cdots & 0 \\
\vdots & \vdots & & \vdots \\
0 & 0 & \cdots & S_{k}
\end{array}\right)
$$

Phase-type distributions with $N=2$ are phase-type distributions with

$$
S=\left(\begin{array}{cc}
-\gamma & \alpha \gamma \\
\beta \mu & -\mu
\end{array}\right),
$$

where $\gamma, \mu>0$ and $0 \leq \alpha, \beta \leq 1, \alpha \beta<1$ with a density satisfying $(7)$, i.e., $f_{X}(x)+b_{1} f_{X}^{\prime}(x)+$ $b_{2} f_{X}^{\prime \prime}(x)=0$.

In [28] it is demonstrated that any phase-type distribution with $N=2$ is either an hyperexponential distribution or a linear combination of an exponential and an $\operatorname{Erlang}(2)$ with the same scalar parameter. Then, following [28], a phase-type distribution with $N=2$, can be always expressed in standardized form with $v=\left(\alpha_{1}, \alpha_{2}\right), S=\left(\begin{array}{cc}-a_{1} & a_{2} \\ 0 & -a_{4}\end{array}\right), a_{1}, a_{2}, a_{4} \geq 0$, being $a_{1}=\beta_{1}$, $a_{4}=\beta_{2}$ and $a_{2}=0$ for the hyper-exponential $\left(\beta_{1}, \beta_{2}\right)$ distribution and $a_{1}=a_{2}=a_{4}=\beta$ for a linear combination of an exponential $(\beta)$ and an $\operatorname{Erlang}(2, \beta)$. It is easy to prove that the following 
relations are fulfilled for the standardized form, $b_{1}=\frac{a_{1}+a_{4}}{a_{1} a_{4}}$ and $b_{2}=\frac{1}{a_{1} a_{4}}$ and that the density function using the standardized form is $f_{X}(x)=\alpha_{1}\left(a_{1}-a_{2}\right) e^{-a_{1} x}+\alpha_{2} a_{4} e^{-a_{4} x}+\alpha_{1} a_{4} a_{2} x e^{-a_{1} x}$.

\section{Gerber-Shiu function with $X$ phase-type 2 distributed}

In order to find the expression of the Gerber-Shiu function if the individual claim amount follows a phase-type distribution we first need to obtain the ordinary differential equation from the integrodifferential equation included in Theorem 1 and then, solve it. In this section, we present some results that are general and useful for any phase-type, the ordinary differential equation and the expression for $\xi_{s}(u)$ for $s=1,2$. Next, we obtain the explicit expression for the Gerber-Shiu function for $N=2$.

\subsection{General results}

In Proposition 1, we present the ordinary differential equation for the Gerber-Shiu function in a Poisson process model. It is general with respect the three specific expressions included in $W D$. This is an important result that implies that if we are analyzing the probability of ruin or the deficit at ruin if ruin occurs, the structure of the solution will be the same. Then, where is the difference? The difference is included in the expression of $\xi_{s}(u)$ for $s=1,2$. In Proposition 2, we present a general expression of $\xi_{s}(u)$.

Proposition 1 If the individual claim amount is distributed as a $P H(v, S)$ and $w(l, j)=w(j)$, $\phi(u)$ is the solution of the ordinary differential equation,

$$
\begin{aligned}
\phi_{s}^{(N+1)}(u) & =\left(\frac{\delta}{c_{s} k_{s}^{N} b_{N}}\right) \phi_{s}(u)+\left(\frac{\lambda+\delta}{c_{s}}-\frac{b_{N-1}}{k_{s} b_{N}}\right) \phi_{s}^{(N)}(u) \\
& -\sum_{j=1}^{N-1} \frac{1}{k_{s}^{N-j}}\left(\frac{\lambda}{c_{s}} f^{(N-1-j)}(0)+\frac{b_{j-1}}{k_{s} b_{N}}-\frac{(\lambda+\delta) b_{j}}{c_{s} b_{N}}\right. \\
& \left.+\frac{\lambda}{c_{s} b_{N}} \sum_{h=j+1}^{N-1} b_{h} f^{(h-j-1)}(0)\right) \phi_{s}^{(j)}(u),
\end{aligned}
$$

where $\phi_{s}(u), s=1,2$ being $s=1$ for $0<u<b$ and $s=2$ for $u>b$.

The proof of Proposition 1 is included in Appendix.

Proposition 2 Let the individual claim amount $X \sim P H(v, S)$. Then

$$
\xi_{s}(u)=H_{s}(u) \cdot G_{s}, \quad s=1,2,
$$

being $H_{s}(u)=\left(1-F_{X_{s}^{*}}(u)\right), S^{*}=\frac{S}{k_{s}}, X_{s}^{*} \sim P H\left(v, S^{*}\right)$ and

$$
G_{s}= \begin{cases}\alpha_{m}(Z) & \text { if } w(l, j)=j^{m}, m \geq 1 \\ F_{Z}(y) & \text { if } w(l, j)=I(j \leq y) \\ 1 & \text { if } w(l, j)=1\end{cases}
$$

for $Z \sim P H\left(v^{*}, S^{*}\right)$ and $v^{*}=\frac{v \exp \left(S^{*} u\right)}{H_{s}(u)}$.

Proof. Taking into account (6), if the penalty function is equal to 1 , assuming that $X \sim P H(v, S)$

$$
\xi_{s}(u)=\int_{\frac{u}{k_{s}}}^{\infty} f(x) d x=v \exp \left(S^{*} u\right) e^{\top}=1-F_{X_{s}^{*}}(u)=H_{s}(u),
$$

where $S^{*}=\frac{S}{k_{s}}$ and $X_{s}^{*} \sim P H\left(v, S^{*}\right)$. 
If the penalty function is $j^{m}$, then

$$
\begin{aligned}
\xi_{s}(u) & =\int_{\frac{u}{k_{s}}}^{\infty}\left(k_{s} x-u\right)^{m} f(x) d x=\int_{0}^{\infty} z^{m} \frac{1}{k_{s}} f\left(\frac{u+z}{k_{s}}\right) d z \\
& =\int_{0}^{\infty} z^{m} v \exp \left(S^{*} u\right) \exp \left(S^{*} z\right)\left(-S^{*} e^{\top}\right) d z, s=1,2 .
\end{aligned}
$$

Let $v^{*}=\frac{v \exp \left(S^{*} u\right)}{H_{s}(u)}$, then

$$
\xi_{s}(u)=H_{s}(u) \int_{0}^{\infty} z^{m} v^{*} \exp \left(S^{*} z\right)\left(-S^{*} e^{\top}\right) d z, s=1,2,
$$

and taking into account that $v^{*} \exp \left(S^{*} z\right)\left(-S^{*} e^{\top}\right)=f_{Z}(z)$ being $Z \sim P H\left(v^{*}, S^{*}\right)$,

$$
\xi_{s}(u)=H_{s}(u)(-1)^{m} m ! v^{*}\left(S^{*}\right)^{-m} e^{\top}=H_{s}(u) \alpha_{m}(Z), s=1,2 .
$$

If the penalty function is $I(j \leq y)$,

$$
\begin{aligned}
\xi_{s}(u) & =\int_{\frac{u}{k_{s}}}^{\frac{u+y}{k_{s}}} d F(x)=1-v \exp \left(S^{*}(u+y)\right) e^{\top}-1+v \exp \left(S^{*} u\right) e^{\top} \\
& =v \exp \left(S^{*} u\right) e^{\top}-v \exp \left(S^{*} u\right) \exp \left(S^{*} y\right) e^{\top}, \quad s=1,2,
\end{aligned}
$$

and considering $v^{*}$ and $H_{s}(u)$,

$$
\xi_{s}(u)=H_{s}(u)\left(1-v^{*} \exp \left(S^{*} y\right) e^{\top}\right)=H_{s}(u) F_{Z}(y), s=1,2 .
$$

\subsection{Results for $N=2$}

From Proposition 2, we derive the following corollary, that gives the expression of $\xi_{s}(u), s=1,2$ assuming a $P H(v, S)$ with $N=2$ expressed in standardized form.

Corollary 1 From Proposition 2, if the individual claim amount is $P H(v, S)$ with $N=2$ expressed in standardized form, being $a_{i, s}$ the elements of $S^{*}=\frac{S}{k_{s}}, s=1,2$,

$$
\xi_{s}(u)=C_{1}^{(s)} e^{-a_{1, s} u}+C_{2}^{(s)} e^{-a_{4, s} u}+C_{3}^{(s)} u e^{-a_{1, s} u}, \quad s=1,2 .
$$

For the penalty functions

i) $w(l, j)=1: C_{1}^{(s)}=\alpha_{1}, C_{2}^{(s)}=\alpha_{2}$ and $C_{3}^{(s)}=\alpha_{1} a_{2, s}$.

ii) $w(l, j)=j^{m}: C_{1}^{(s)}=\alpha_{1}\left(\frac{m !}{a_{1, s}^{m}}+m ! \frac{a_{2, s}}{a_{1, s}^{m+1}} \sum_{i=1}^{m} a_{1, s}^{i} a_{4, s}^{-i}\right), C_{2}^{(s)}=\alpha_{2} \frac{m !}{a_{4, s}^{m}}$ and $C_{3}^{(s)}=$ $\alpha_{1} a_{2, s} \frac{m !}{a_{4, s}^{m}}$.

iii) $w(l, j)=I(j \leq y): C_{1}^{(s)}=\alpha_{1}\left(1-e^{-a_{1, s} y}\right), C_{2}^{(s)}=\alpha_{2}\left(1-e^{-a_{4, s} y}\right)-\alpha_{1} a_{2, s} y e^{-a_{1, s} y}$ and $C_{3}^{(s)}=\alpha_{1} a_{2, s}\left(1-e^{-a_{1, s} y}\right)$.

Proof. Let $X \sim P H(v, S)$, be $S$ a matrix expressed in standardized form, and be $a_{i, s}$ the elements of $S^{*}=\frac{S}{k_{s}}, s=1,2$.

For $w(l, j)=1$, from (11), if we let $a_{i, s}=\frac{a_{i}}{k_{s}}$,

$$
\begin{aligned}
\xi_{s}(u) & =\left(\alpha_{1}, \alpha_{2}\right)\left(\begin{array}{cc}
e^{-a_{1, s} u} & a_{2, s} u e^{-a_{1, s} u} \\
0 & e^{-a_{4, s} u}
\end{array}\right) e^{\top} \\
& =\alpha_{1} e^{-a_{1, s} u}+\alpha_{2} e^{-a_{4, s} u}+\alpha_{1} a_{2, s} u e^{-a_{1, s} u} .
\end{aligned}
$$


For $w(l, j)=j^{m}$, from (13), and substituting $v^{*}=\frac{v \exp \left(S^{*} u\right)}{H_{s}(u)}$,

$$
\begin{aligned}
\xi_{s}(u) & =m !(-1)^{m} v \exp \left(S^{*} u\right)\left(S^{*}\right)^{-m} e^{\top} \\
& =m !(-1)^{m}\left(\alpha_{1}, \alpha_{2}\right)\left(\begin{array}{cc}
e^{-a_{1, s} u} & a_{2, s} u e^{-a_{1, s} u} \\
0 & e^{-a_{4, s} u}
\end{array}\right)\left(\begin{array}{cc}
-a_{1, s} & a_{2, s} \\
0 & -a_{4, s}
\end{array}\right)^{-m} e^{\top},
\end{aligned}
$$

being

$$
\left(\begin{array}{cc}
-a_{1} & a_{2} \\
0 & -a_{4}
\end{array}\right)^{-m}=(-1)^{m}\left(\begin{array}{cc}
\frac{1}{a_{1, s}^{m}} & \frac{a_{2, s}}{a_{1, s}^{m+1}} \sum_{i=1}^{m} a_{1, s}^{i} a_{4, s}^{-i} \\
0 & \frac{1}{a_{4, s}^{m}}
\end{array}\right)
$$

then

$$
\begin{aligned}
\xi_{s}(u) & =\alpha_{1}\left(\frac{m !}{a_{1, s}^{m}}+m ! \frac{a_{2, s}}{a_{1, s}^{m+1}} \sum_{i=1}^{m} a_{1, s}^{i} a_{4, s}^{-i}\right) e^{-a_{1, s} u} \\
& +\alpha_{2} \frac{m !}{a_{4, s}^{m}} e^{-a_{4, s} u}+\alpha_{1} \frac{m !}{a_{4, s}^{m}} a_{2, s} u e^{-a_{1, s} u} .
\end{aligned}
$$

And for $w(l, j)=I(j \leq y)$, from (15) and using $v^{*}$,

$$
\begin{aligned}
\xi_{s}(u) & =v \exp \left(S^{*} u\right) e^{\top}-v \exp \left(S^{*} y\right) \exp \left(S^{*} u\right) e^{\top} \\
& =\alpha_{1}\left(1-e^{-a_{1, s} y}\right) e^{-a_{1, s} u}+\left(\alpha_{2}\left(1-e^{-a_{4, s} y}\right)-\alpha_{1} a_{2, s} y e^{-a_{1, s} y}\right) e^{-a_{4, s} u} \\
& +\alpha_{1}\left(1-e^{-a_{1, s} y}\right) a_{2, s} u e^{-a_{1, s} u} .
\end{aligned}
$$

Then, the corollary is proved.

Obviously, from Corollary 1 , it is possible to obtain the particular cases included in phase-type 2 distributions. If we consider the hyper-exponential $\left(\beta_{1}, \beta_{2}\right)$ distribution, $a_{1}=\beta_{1}, a_{2}=0$ and $a_{4}=\beta_{2}$. Then, for the penalty function equal to $1, C_{1}^{(s)}=\alpha_{1}, C_{2}^{(s)}=\alpha_{2}$ and $C_{3}^{(s)}=0$; for the penalty function equal to $j^{m}, C_{1}^{(s)}=\alpha_{1} \frac{k_{s}^{m} m !}{\beta_{1}^{m}}, C_{2}^{(s)}=\alpha_{2} \frac{k_{s}^{m} m !}{\beta_{2}^{m}}$ and $C_{3}^{(s)}=0$; and for penalty function equal to $I(j \leq y), C_{1}^{(s)}=\alpha_{1}\left(1-e^{-\frac{\beta_{1}}{k_{s}} y}\right), C_{2}^{(s)}=\alpha_{2}\left(1-e^{-\frac{\beta_{2}}{k_{s}} y}\right)$ and $C_{3}^{(s)}=0$.

If we consider a linear combination of an $\operatorname{exponential}(\beta)$ and an $\operatorname{Erlang}(2, \beta), a_{1}=a_{2}=a_{4}=\beta$. Then, for the penalty function equal to $1, C_{1}^{(s)}=\alpha_{1}, C_{2}^{(s)}=\alpha_{2}$ and $C_{3}^{(s)}=\alpha_{1} \frac{\beta}{k_{s}}$; for the penalty function equal to $j^{m}, C_{1}^{(s)}=\alpha_{1} \frac{k_{s}^{m}(m+1) !}{\beta^{m}}, C_{2}^{(s)}=\alpha_{2} \frac{k_{s}^{m} m !}{\beta^{m}}$ and $C_{3}^{(s)}=\alpha_{1} \frac{k_{s}^{m-1} m !}{\beta^{m-1}}$; and for the penalty function equal to $I(j \leq y), C_{1}^{(s)}=\alpha_{1}\left(1-e^{-\frac{\beta}{k_{s}} y}\right), C_{2}^{(s)}=\alpha_{2}\left(1-e^{-\frac{\beta}{k_{s}} y}\right)-\alpha_{1} \frac{\beta}{k_{s}} y e^{-\frac{\beta}{k_{s}} y}$ and $C_{3}^{(s)}=\alpha_{1} \frac{\beta}{k_{s}}\left(1-e^{-\frac{\beta}{k_{s}} y}\right)$. If, in addition, we consider $\alpha_{1}=1$ and $\alpha_{2}=0$, then we get the Erlang $(2, \beta)$ distribution. The exponential distribution is not a phase-type 2 , but it can be obtained considering that $\alpha_{1}=0$ and $\alpha_{2}=1$.

Once we have obtained the different expressions of $\xi_{s}(u)$, we can solve the integro-differential equation for the Gerber-Shiu function. From (10), if $N=2$,

$$
\begin{aligned}
\phi_{s}^{\prime \prime \prime}(u) & =\left(\frac{\lambda+\delta}{c_{s}}-\frac{b_{1}}{k_{s} b_{2}}\right) \phi_{s}^{\prime \prime}(u) \\
& +\left(\frac{(\lambda+\delta) b_{1}}{c_{s} k_{s} b_{2}}-\frac{b_{0}}{k_{s}^{2} b_{2}}-\frac{\lambda}{c_{s} k_{s}} f(0)\right) \phi_{s}^{\prime}(u)+\frac{\delta}{c_{s} k_{s}^{2} b_{2}} \phi_{s}(u),
\end{aligned}
$$

where $\phi_{s}(u), s=1,2$ being $s=1$ for $0<u<b$ and $s=2$ for $u>b$.

In order to solve (16) we obtain the characteristic equation for $0 \leq u<b$,

$$
r^{3}-\left(\frac{\lambda+\delta}{c_{1}}-\frac{b_{1}}{k_{1} b_{2}}\right) r^{2}-\left(\frac{(\lambda+\delta) b_{1}}{c_{1} k_{1} b_{2}}-\frac{b_{0}}{k_{1}^{2} b_{2}}-\frac{\lambda}{c_{1} k_{1}} f(0)\right) r-\frac{\delta}{c_{1} k_{1}^{2} b_{2}}=0,
$$


and for $u \geq b$,

$$
r^{3}-\left(\frac{\lambda+\delta}{c_{2}}-\frac{b_{1}}{k_{2} b_{2}}\right) r^{2}-\left(\frac{(\lambda+\delta) b_{1}}{c_{2} k_{2} b_{2}}-\frac{b_{0}}{k_{2}^{2} b_{2}}-\frac{\lambda}{c_{2} k_{2}} f(0)\right) r-\frac{\delta}{c_{2} k_{2}^{2} b_{2}}=0,
$$

with $r_{i}, i=1, \ldots, 6$, real and distinct roots of the characteristic equations, so

$$
\phi(u)=\left\{\begin{array}{l}
\phi_{1}(u)=\sum_{i=1}^{3} D_{i} e^{r_{i} u}, 0 \leq u<b, \\
\phi_{2}(u)=\sum_{i=4}^{6} D_{i} e^{r_{i} u}, \quad u \geq b .
\end{array}\right.
$$

To obtain the ruin probability, and the moments and the deficit at ruin (not their present values) we have to consider $\delta=0$, so $r_{3}=r_{6}=0$.

Then, to determine $D_{i}, i=1, \ldots, 6$, we need six equations. One equation is obtained from $\lim _{u \rightarrow \infty} \phi(u)=0$, that gives $D_{6}=0$. Another equation comes from the continuity condition

$$
\left.\phi_{1}(u)\right|_{u=b^{-}}=\phi_{2}(b) .
$$

The other four conditions are obtained substituting (17) in (5), integrating and rearranging terms, considering Corollary 1 , and taking into account the values of $a_{1}, a_{2}$ and $a_{4}$.

For the hyper-exponential $\left(\beta_{1}, \beta_{2}\right)$ distribution, let us define $h_{1}=\frac{C_{1}^{(1)}}{\alpha_{1}}, h_{2}=\frac{C_{2}^{(1)}}{\alpha_{2}}, h_{3}=\frac{C_{1}^{(2)}}{\alpha_{1}}$ and $h_{4}=\frac{C_{2}^{(2)}}{\alpha_{2}}$, then the four equations are

$$
\begin{gathered}
\beta_{1} \sum_{i=1}^{3} \frac{D_{i}}{r_{i} k_{1}+\beta_{1}}=h_{1}, \\
\beta_{2} \sum_{i=1}^{3} \frac{D_{i}}{r_{i} k_{1}+\beta_{2}}=h_{2}, \\
\beta_{1} \sum_{i=4}^{5} \frac{D_{i} e^{\left(r_{i}+\frac{\beta_{1}}{k_{2}}\right)^{b}}}{r_{i} k_{2}+\beta_{1}}+\beta_{1} \sum_{i=1}^{3} \frac{D_{i}\left(1-e^{\left.\left(r_{i}+\frac{\beta_{1}}{k_{2}}\right)^{b}\right)}\right.}{r_{i} k_{2}+\beta_{1}}=h_{3}, \\
\beta_{2} \sum_{i=4}^{5} \frac{D_{i} e^{\left(r_{i}+\frac{\beta_{2}}{k_{2}}\right)^{b}}}{r_{i} k_{2}+\beta_{2}}+\beta_{2} \sum_{i=1}^{3} \frac{D_{i}\left(1-e^{\left(r_{i}+\frac{\beta_{2}}{k_{2}}\right)^{b}}\right)}{r_{i} k_{2}+\beta_{2}}=h_{4} .
\end{gathered}
$$

For the linear combination of an $\operatorname{exponential}(\beta)$ and an $\operatorname{Erlang}(2, \beta)$, let us define $h_{1}=C_{1}^{(1)}+C_{2}^{(1)}$, $h_{2}=\frac{C_{3}^{(1)} k_{1}}{\alpha_{1} \beta}, h_{3}=C_{1}^{(2)}+C_{2}^{(2)}$ and $h_{4}=\frac{C_{3}^{(2)} k_{2}}{\alpha_{1} \beta}$, then the four equations are

$$
\begin{gathered}
\sum_{i=1}^{3} \frac{\alpha_{1} D_{i} \beta^{2}}{\left(r_{i} k_{1}+\beta\right)^{2}}+\sum_{i=1}^{3} \frac{\alpha_{2} D_{i} \beta}{r_{i} k_{1}+\beta}=h_{1}, \\
\sum_{i=1}^{3} \frac{D_{i} \beta}{r_{i} k_{1}+\beta}=h_{2}, \\
\left(\alpha_{2} \beta-\frac{\alpha_{1} \beta^{2} b}{k_{2}}\right) \sum_{i=4}^{5} \frac{D_{i} e^{\left(r_{i}+\frac{\beta}{k_{2}}\right)^{b}}}{r_{i} k_{2}+\beta}+\alpha_{1} \beta^{2} \sum_{i=4}^{5} \frac{D_{i} e^{\left(r_{i}+\frac{\beta}{k_{2}}\right)^{b}}}{\left(r_{i} k_{2}+\beta\right)^{2}}+\frac{\alpha_{1} \beta^{2} b}{k_{2}} \sum_{i=1}^{3} \frac{D_{i} e^{\left(r_{i}+\frac{\beta}{k_{2}}\right) b}}{r_{i} k_{2}+\beta} \\
+\alpha_{1} \beta^{2} \sum_{i=1}^{3} \frac{D_{i}\left(1-e^{\left(r_{i}+\frac{\beta}{k_{2}}\right) b}\right)}{\left(r_{i} k_{2}+\beta\right)^{2}}+\alpha_{2} \beta \sum_{i=1}^{3} \frac{D_{i}\left(1-e^{\left(r_{i}+\frac{\beta}{k_{2}}\right) b}\right)}{r_{i} k_{2}+\beta}=h_{3}, \\
\beta \sum_{i=4}^{5} \frac{D_{i} e^{\left(r_{i}+\frac{\beta}{k_{2}}\right) b}}{r_{i} k_{2}+\beta}+\beta \sum_{i=1}^{3} \frac{D_{i}\left(1-e^{\left.\left(r_{i}+\frac{\beta}{k_{2}}\right)^{b}\right)}\right.}{r_{i} k_{2}+\beta}=h_{4} .
\end{gathered}
$$


Let us rewrite the linear equation system formed by (18) and (19) or (20) in matrix form, $A \cdot D=H$, being $D$ the vector of unknowns, $D=\left(D_{j}\right)_{j=1, \ldots, 5}$, considering $H$ the vector of independent terms $H=\left(0, h_{1}, h_{2}, h_{3}, h_{4}\right)^{\top}$, and $A$ the matrix of the coefficients of the system.

Solving the system we obtain $D=A^{-1} H$, so $D_{j}=\sum_{i=1}^{4} h_{i} \cdot f_{j i}, j=1, \ldots, 5$, being $f_{j i}$ the elements of the matrix $A^{-1}$. From (17),

$$
\phi(u)=\left\{\begin{array}{l}
\phi_{1}(u)=\sum_{i=1}^{3} D_{i} e^{r_{i} u}=\sum_{z=1}^{4} h_{z} \cdot \sum_{i=1}^{3} f_{i z} e^{r_{i} u}=\sum_{z=1}^{4} h_{z} \cdot c_{z}(u), 0 \leq u<b, \\
\phi_{2}(u)=\sum_{i=4}^{5} D_{i} e^{r_{i} u}=\sum_{z=1}^{4} h_{z} \cdot \sum_{i=4}^{5} f_{i z} e^{r_{i} u}=\sum_{z=1}^{4} h_{z} \cdot d_{z}(u), \quad u \geq b .
\end{array}\right.
$$

being $c_{z}(u)=\sum_{i=1}^{3} f_{i z} e^{r_{i} u}$ and $d_{z}(u)=\sum_{i=4}^{5} f_{i z} e^{r_{i} u}$.

From the definition of $h_{z}, z=1, \ldots, 4$, it is straightforward that in the ruin probability case, $h_{z}=1$. Then, from $(21), \phi_{1}(u)=\psi_{1}(u)=\sum_{z=1}^{4} c_{z}(u)$ and $\phi_{2}(u)=\psi_{2}(u)=\sum_{z=1}^{4} d_{z}(u)$.

Proposition 3 The deficit at ruin if ruin occurs, $Y$, is distributed as a phase-type $P H(\tau(u), M)$ where $\tau(u)=\left(P 1_{z}(u)\right)_{z=1, \ldots, 4}$ being $P 1_{z}(u)=\frac{c_{z}(u)}{\psi_{1}(u)}$ if $0 \leq u<b$, and $\tau(u)=\left(P 2_{z}(u)\right)_{z=1, \ldots, 4}$ being $P 2_{z}(u)=\frac{d_{z}(u)}{\psi_{2}(u)}$ if $u \geq b$, and

$$
M=\left(\begin{array}{cc}
Q_{1} & \mathbf{0} \\
\mathbf{0} & Q_{2}
\end{array}\right)
$$

being $Q_{s}=\left(\begin{array}{cc}-\frac{a_{1}}{k_{s}} & \frac{\alpha_{1} a_{2}}{k_{s}} \\ 0 & -\frac{a_{4}}{k_{s}}\end{array}\right), s=1,2$.

Proof. The distribution of the deficit at ruin if ruin occurs from $(3)$ and $\delta=0$ is $F_{Y}(y)=\frac{\phi(u)}{\psi(u)}$. For $0 \leq u<b$, from $(21)$,

$$
F_{Y}(y)=\frac{\phi_{1}(u)}{\psi_{1}(u)}=\frac{1}{\psi_{1}(u)} \sum_{z=1}^{4} h_{z} \cdot c_{z}(u)=\sum_{z=1}^{4} h_{z} \cdot P 1_{z}(u) .
$$

Knowing the values of $h_{z}, z=1, \ldots, 4$, defined in (19) and (20), and considering the values of $C_{i}^{(s)}$ in Corollary 1 for $w(l, j)=I(j \leq y)$, substituting in (22), and grouping terms we obtain

$$
\begin{aligned}
F_{Y}(y) & =1-W_{1}(u)\left(\frac{P 1_{1}(u)}{W_{1}(u)}, \frac{P 1_{2}(u)}{W_{1}(u)}\right) \exp \left(Q_{1} y\right) e^{\top} \\
& -W_{2}(u)\left(\frac{P 1_{3}(u)}{W_{2}(u)}, \frac{P 1_{4}(u)}{W_{2}(u)}\right) \exp \left(Q_{2} y\right) e^{\top},
\end{aligned}
$$

being

$$
\begin{aligned}
& W_{1}(u)=P 1_{1}(u)+P 1_{2}(u), \\
& W_{2}(u)=P 1_{3}(u)+P 1_{4}(u), \\
& Q_{s}=\left(\begin{array}{cc}
-\frac{a_{1}}{k_{s}} & -\frac{\alpha_{1} a_{2}}{k_{s}} \\
0 & -\frac{a_{4}}{k_{s}}
\end{array}\right) .
\end{aligned}
$$

Let $v_{1}=\left(\frac{P 1_{1}(u)}{W_{1}(u)}, \frac{P 1_{2}(u)}{W_{1}(u)}\right)$ and $v_{2}=\left(\frac{P 1_{3}(u)}{W_{2}(u)}, \frac{P 1_{4}(u)}{W_{2}(u)}\right)$, then $(23)$ can be written as

$$
F_{Y}(y)=1-W_{1}(u) v_{1} \exp \left(Q_{1} y\right) e^{\top}-W_{2}(u) v_{2} \exp \left(Q_{2} y\right) e^{\top} .
$$

Taking into account that $Q_{s}, s=1,2$ has the structure defined in (9), then the distribution of the deficit at ruin if ruin occurs is a mixture of two phase-type distributions, $Y_{1} \sim P H\left(v_{1}, Q_{1}\right)$ and 
$Y_{2} \sim P H\left(v_{2}, Q_{2}\right)$, being the weights $W_{1}(u)$ and $W_{2}(u)$. Then, considering that the finite mixture of a phase-type distribution is a phase-type distribution, from (8), the proposition for $0 \leq u<b$ is proved.

For $u \geq b$, applying a similar process it can be demonstrated that $Y \sim P H(\tau(u), M)$, with $\tau(u)=\left(P 2_{z}(u)\right)_{z=1, \ldots, 4}$. So, $Y$ is a mixture of two phase-type distributions $Y_{1} \sim P H\left(v_{3}, Q_{1}\right)$ and $Y_{2} \sim P H\left(v_{4}, Q_{2}\right)$ with $v_{3}=\left(\frac{P 2_{1}(u)}{V_{1}(u)}, \frac{P 2_{2}(u)}{V_{1}(u)}\right)$ and $v_{4}=\left(\frac{P 2_{3}(u)}{V_{2}(u)}, \frac{P 2_{4}(u)}{V_{2}(u)}\right)$, being the weights

$$
V_{1}(u)=\left(P 2_{1}(u)+P 2_{2}(u)\right) \text { and } V_{2}(u)=\left(P 2_{3}(u)+P 2_{4}(u)\right) .
$$

Example 1 As an example, we calculate the probabilities of ruin and the distribution of the deficit at ruin if ruin occurs assuming a threshold reinsurance strategy with $X_{i} \sim \operatorname{Erlang}(2, \beta)$ and the following values for the parameters $\beta=2, \lambda=1, b=2, k_{1}=0.8, k_{2}=0.45, \rho=0.15, \rho_{R}=0.25$ and $\delta=0$.

Let us first obtain the ruin probability. We know that, in this case, the independent terms of system (20), $h_{z}, z=1, \ldots, 4$ are equal to one and that the matrix $A^{-1}$ is

$$
A^{-1}=\left(\begin{array}{rrrrr}
0.15396 & -0.16072 & 1.632 \times 10^{-5} & 1.6139 \times 10^{-4} & 0.24325 \\
0.1452 & 0.34836 & -1.1930 \times 10^{-3} & -1.1797 \times 10^{-2} & -17.781 \\
0.16344 & 0.28890 & 1.3237 \times 10^{-3} & 0.01309 & 19.73 \\
29.622 & -74.895 & 8.8605 & 60.694 & -66773 \\
0.30913 & 0.62036 & 5.7433 \times 10^{-4} & 5.7328 \times 10^{-3} & 7.7797
\end{array}\right)
$$

Then, we have

$$
\begin{aligned}
& \psi_{1}(u)=0.466753-0.0065744 e^{-3.70127 u}+0.480572 e^{-0.187624 u} \\
& \psi_{2}(u)=24.2807 e^{-6.6464 u}+0.935799 e^{-0.0803242 u}
\end{aligned}
$$

Let us know consider the deficit at ruin if ruin occurs. From Proposition 3, $Y$ is distributed as a phase-type, $P H(\tau(u), M)$, with

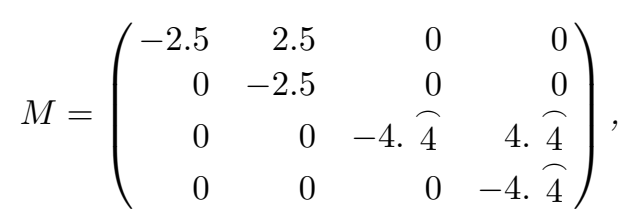

$\tau(u)=$

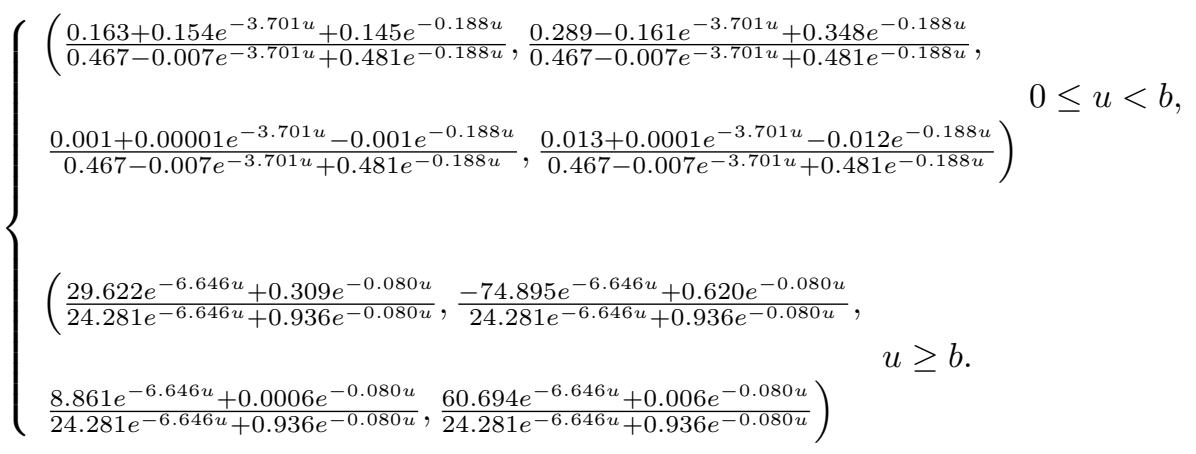


For example, for $u=0$,

$$
\begin{gathered}
\psi_{1}(0)=0.94075, \\
\tau(0)=\left(0.49174,0.50655,1.563 \times 10^{-4}, 1.546 \times 10^{-3}\right), \\
F_{Y}(y)=1-(0.99829+1.22935 y) e^{-2.5 y}-(0.00170244+0.000694874 y) e^{-4 . \widehat{4} y}, \\
\alpha_{m}(Y)=\left(1.49004 \times 0.4^{m}+0.00185879 \times 0.225^{m}\right) m !
\end{gathered}
$$

and for $u=3$,

$$
\begin{gathered}
\psi_{2}(3)=0.740473 \\
\tau(3)=(0.33034,0.66292,0.000613754,0.00612626), \\
F_{Y}(y)=1-(0.99326+0.825849 y) e^{-2.5 y}-(0.00674+0.0027278 y) e^{-4 . \widehat{4} y}, \\
\alpha_{m}(Y)=\left(1.3236 \times 0.4^{m}+0.00735376 \times 0.225^{m}\right) m !
\end{gathered}
$$

\section{Influence of (threshold) proportional reinsurance on the deficit at ruin if ruin occurs}

In this section, we quantify the effect on the deficit at ruin if ruin occurs of a proportional reinsurance (included the threshold). It is known, [29], that when the individual claim amount follows a phase-type distribution $P H(v, S)$, the deficit at ruin if ruin occurs, $Y$, is also phase-type distributed with representation $P H\left(\Pi_{G}, S\right)$, where

$$
\Pi_{G}=\frac{v_{+} \exp (u B)}{\psi(u)},
$$

with $B=S+D, D=S^{0} v_{+}, S^{0}=-S e^{\top}$ being $v_{+}=-\frac{\lambda}{c} v S^{-1}$, in the Poisson case. We also have $\psi(u)=v_{+} \exp (u B) e^{\top}$.

Then, if the insurer uses a proportional reinsurance contract to reduce the risk, what is the effect on the probability of ruin and on the deficit at ruin if ruin occurs? Let us consider a proportional reinsurance with parameter $k, 0<k \leq 1$, such that the retained claim amount for the insurer is $X_{R}=k X$ and the retained premium is $\lambda E[X](1+\rho)-\lambda E[X](1-k)\left(1+\rho_{R}\right)$. We consider that the retention level $k$ gives new positive security loading for the insurer, $\rho_{N}=\frac{\rho-\rho_{R}(1-k)}{k}>0$, i.e., the net profit condition is always fulfilled (see [30] for more details). Then, if $X$ follows a phase-type distribution $P H(v, S), X_{R}$ is also phase-type distributed, $P H\left(v, \frac{S}{k}\right)$. The ruin probability with proportional reinsurance is

$$
\psi(u)=\frac{v S^{-1} \exp \left(u B_{R}\right) e^{\top}}{v S^{-1} e^{\top}\left(1+\rho_{N}\right)}
$$

being $B_{R}=\frac{S}{k}-\frac{S}{k} e^{\top} \frac{v S^{-1}}{v S^{-1} e^{\top}\left(1+\rho_{N}\right)}$. The deficit at ruin if ruin occurs, $Y$, is phase-type distributed, $P H\left(\Pi_{R G}, \frac{S}{k}\right)$, where

$$
\Pi_{R G}=\frac{v S^{-1} \exp \left(u B_{R}\right)}{v S^{-1} \exp \left(u B_{R}\right) e^{\top}} .
$$

Hence, the expectation and the variance of the deficit at ruin if ruin occurs can be easily calculated: $E[Y]=-\Pi_{R G} k S^{-1} e^{\top}$ and $V[Y]=2 \Pi_{R G} k^{2} S^{-2} e^{\top}-\left(\Pi_{R G} k S^{-1} e^{\top}\right)^{2}$. 
The Value at Risk of $Y$ at level $p, \operatorname{VaR}_{p}[Y]$ is such that $F_{Y}\left(\operatorname{VaR}_{p}[Y]\right)=p$, that is $\operatorname{VaR}_{p}[Y]=F_{Y}^{-1}(p)$. However, there is no explicit expression for this $\operatorname{VaR}_{p}[Y]$, it has to be calculated numerically (the package actuar in [31] provides functions for phase-type distributions). The Tail Value at Risk of $Y$ at level $p, T V a R_{p}[Y]$, can be calculated from the Value at Risk at the same level considering the following formula [32],

$$
T V a R_{p}[Y]=\operatorname{VaR}_{p}[Y]-\frac{\Pi_{R G} S^{-1} \exp \left(\operatorname{VaR}_{p}[Y] \frac{S}{k}\right) e^{\top}}{\Pi_{R G} \exp \left(\operatorname{VaR}_{p}[Y] \frac{S}{k}\right) e^{\top}}
$$

As an application, we develop the example that has first been used in [33]. They consider an individual claim amount distribution that is an equal mixture of two exponentials at rates 3 and 7 respectively, with Poisson claims at rate $\lambda=1$ and a relative security loading $\rho=0.4$. In this case, $X$ is $P H(v, S)$, where $v=(0.5,0.5), S=\left(\begin{array}{rr}-3 & 0 \\ 0 & -7\end{array}\right)$ and $B=\left(\begin{array}{rr}-\frac{3}{2} & \frac{9}{14} \\ \frac{7}{2} & -\frac{11}{2}\end{array}\right)$. The ruin probability is $\psi(u)=\frac{24 e^{-u}+e^{-6 u}}{35}$. The deficit at ruin if ruin occurs, $Y$, is phase-type distributed, $P H\left(\Pi_{G}, S\right)$, where $\Pi_{G}=\left(\frac{42-7 e^{-5 u}}{48+2 e^{-5 u}}, \frac{6+9 e^{-5 u}}{48+2 e^{-5 u}}\right)$, being

$$
F_{Y}(y)=1-\frac{6 e^{5 u-7 y}+42 e^{5 u-3 y}+9 e^{-7 y}-7 e^{-3 y}}{2+48 e^{5 u}}
$$

and

$$
\begin{aligned}
& E[Y]=\frac{156-11 e^{-5 u}}{21 e^{-5 u}+504}, \\
& V[Y]=\frac{26352-383 e^{2(-5 u)}-744 e^{-5 u}}{441 e^{2(-5 u)}+21168 e^{-5 u}+254016} .
\end{aligned}
$$

It is straightforward to include in the model a proportional reinsurance. Let consider a retention level $k$ and a security loading of the reinsurer $\rho_{R}=0.5$, with $0.2<k \leq 1$. Then, the net security loading for the insurer is $\rho_{N}=\frac{0.5 k-0.1}{k}$. The ruin probability is

$$
\psi(u)=\frac{e^{\frac{(5-54 k+N) u}{k(-1+15 k)} k\left(-4+165 k+5 N+e^{\frac{2 N u}{k-15 k^{2}}}(4-165 k+5 N)\right)}}{(-1+15 k) N}
$$

being $N=\sqrt{4-120 k+1341 k^{2}}$.

The deficit at ruin if ruin occurs, $Y$, is phase-type distributed, $P H\left(\Pi_{R G}, \frac{S}{k}\right)$, with

$$
\begin{aligned}
\Pi_{R G}= & \frac{7\left(-2+51 k+N+e^{\frac{2 N u}{k-15 k^{2}}}(2-51 k+N)\right)}{2\left(-4+165 k+5 N+e^{\frac{2 N u}{k-15 k^{2}}}(4-165 k+5 N)\right)}, \\
& \left.\frac{3\left(2-9 k+N+e^{\frac{2 N u}{k-15 k^{2}}}(-2+9 k+N)\right)}{2\left(-4+165 k+5 N+e^{\frac{2 N u}{k-15 k^{2}}}(4-165 k+5 N)\right)}\right)
\end{aligned}
$$


and

$$
\begin{aligned}
E[Y] & =-\frac{k\left(40-1209 k+e^{\frac{2 N u}{k-15 k^{2}}}(-40+1209 k-29 N)-29 N\right)}{21\left(-4+165 k+5 N+e^{\frac{2 N u}{k-15 k^{2}}}(4-165 k+5 N)\right)}, \\
V[Y]= & \frac{2 k^{2}\left(-84 e^{\frac{2 N u}{k-15 k^{2}}}\left(530 k+375 k^{2}-37\right)+M-1160 N+k(38589 N-98820)\right)}{441\left(-4+165 k+5 N+e^{\frac{2 N u}{k-15 k^{2}}}(4-165 k+5 N)\right)^{2}} \\
& +\frac{2 k^{2}\left(e^{\frac{4 N u}{k-15 k^{2}}}(M+1160 N-3 k(32940+12863 N))\right)}{441\left(-4+165 k+5 N+e^{\frac{2 N u}{k-15 k^{2}}}(4-165 k+5 N)\right)^{2}},
\end{aligned}
$$

being $M=2482+1368819 k^{2}$.

We consider now a threshold proportional reinsurance, that is defined by its three parameters (b, $k_{1}$ and $k_{2}$ ). In this case, the probability of ruin is given by (21), and the deficit at ruin if ruin occurs is phase-type 4 distributed (see Proposition 3). The explicit expressions of the probability of ruin and the different measures of the deficit at ruin if ruin occurs can then be easily obtained (see Example 1 for the $\operatorname{Erlang}(2, \beta)$ ).

Optimization problems regarding ruin probability. Which is the best strategy in order to minimize the ruin probability of the insurer? In order to answer this question we solve two optimization problems. Firstly, the insurer only considers the proportional reinsurance option. Let $\psi_{k}(u)$ be the ruin probability when all the variables that influence the probability are fixed except the retention level $k$,

$$
\begin{aligned}
& \min _{k,} \psi_{k}(u) \\
& 0.2<k \leq 1
\end{aligned}
$$

being (24) the expression for the probability of ruin in this case. It can be proved that this optimum exists, but the expressions for the optimal point and the minimum value have not been included for the sake of brevity. In Table 1, we include the results of this minimization for different values of $u$, being $k^{*}$ the minimum point. The expectation, the variance and the Value at Risk and the Tail Value at Risk for different levels $p(0.95,0.99$ and 0.995$)$ of the deficit at ruin if ruin occurs can also be found in Table 1 for the optimal $k^{*}$.

Tabla 1. Minimum probabilities of ruin with proportional reinsurance and $E[Y], V[Y], \operatorname{VaR}_{p}[Y]$ and $T V a R_{p}[Y]$.

\begin{tabular}{rrccccccccc}
\hline \multicolumn{1}{c}{$u$} & \multicolumn{1}{c}{$k^{*}$} & $\psi_{k^{*}}(u)$ & $E[Y]$ & $V[Y]$ & $V a R_{0.95}[Y]$ & $T V a R_{0.95}[Y]$ & $V a R_{0.99}[Y]$ & $T V a R_{0.99}[Y]$ & $V a R_{0.995}[Y]$ & $T V a R_{0.995}[Y]$ \\
\hline 0 & 1 & 0.714286 & 0.276 & 0.0915 & 0.883824 & 1.214810 & 1.416660 & 1.749710 & 1.647410 & 1.980630 \\
0.25 & 0.466294 & 0.497108 & 0.143 & 0.0223 & 0.442170 & 0.597268 & 0.691811 & 0.847203 & 0.799507 & 0.954922 \\
0.50 & 0.407213 & 0.321745 & 0.125 & 0.0171 & 0.387419 & 0.522888 & 0.605465 & 0.741171 & 0.699518 & 0.835243 \\
1 & 0.381941 & 0.132298 & 0.117 & 0.0150 & 0.363249 & 0.490308 & 0.567759 & 0.695043 & 0.655975 & 0.783277 \\
2 & 0.370573 & 0.022125 & 0.114 & 0.0141 & 0.352356 & 0.475633 & 0.550778 & 0.674273 & 0.636367 & 0.759880 \\
3 & 0.366956 & 0.003691 & 0.113 & 0.0139 & 0.348890 & 0.470963 & 0.545374 & 0.667664 & 0.630129 & 0.752436 \\
5 & 0.364121 & 0.000103 & 0.112 & 0.0136 & 0.346174 & 0.467303 & 0.541139 & 0.662484 & 0.625239 & 0.746601 \\
\hline
\end{tabular}

Source: Own elaboration

Table 1 shows that the minimum ruin probability and the optimal retention level decrease as the initial reserves are increased. Considering that the insurer retains precisely that optimal level 
that minimizes the ruin probability, the measures analyzed in the table show a decrease in all cases when the initial reserves are higher. As a particular case, the results show that if the insurer has zero initial reserves, the best option is not to reinsure $\left(k^{*}=1\right)$ and to retain all the business (this result is consistent with the one obtained in [4]).

The second optimization problem appears when the insurer considers the threshold proportional reinsurance option (then, (25) is a particular case). Let $\psi\left(b, k_{1}, k_{2}\right)$ be the ruin probability as a function of $\left(b, k_{1}, k_{2}\right)$ when $u$ is considered to be a parameter. Thus, the problem is,

$$
\begin{gathered}
\min _{b, k_{1}, k_{2},} \psi\left(b, k_{1}, k_{2}\right) \\
0.2<k_{1} \leq 1 \\
0.2<k_{2} \leq 1
\end{gathered}
$$

where the ruin probability is calculated with (21). This problem is solved numerically with the function NMinimize of the software Mathematica. Table 2 includes the optimum $\left(b^{*}, k_{1}^{*}, k_{2}^{*}\right)$ with the corresponding minimum probability of ruin for different values of $u$. We have also included $E[Y], V[Y], \operatorname{VaR}_{p}[Y]$ and $T V a R_{p}[Y]$ as in Table 1 for proportional reinsurance.

Tabla 2. Minimum probabilities of ruin with threshold proportional reinsurance and $E[Y], V[Y], V a R_{p}[Y]$ and $T \operatorname{TaR} R_{p}[Y]$.

\begin{tabular}{rcccccccccc}
\hline \multicolumn{1}{c}{$u$} & $\left(b^{*}, k_{1}^{*}, k_{2}^{*}\right)$ & $\psi\left(b^{*}, k_{1}^{*}, k_{2}^{*}\right)$ & $E[Y]$ & $V[Y]$ & $V a R_{0.95}[Y]$ & $T V a R_{0.95}[Y]$ & $V a R_{0.99}[Y]$ & $T V a R_{0.99}[Y]$ & $V a R_{0.995}[Y]$ & $T V a R_{0.995}[Y]$ \\
\hline 0 & $(0.403113,1,0.35665)$ & 0.645002 & 0.25746 & 0.08426 & 0.839819 & 1.16940 & 1.37048 & 1.70337 & 1.60106 \\
0.25 & $(0.403113,1,0.35665)$ & 0.428963 & 0.26051 & 0.08640 & 0.851860 & 1.18255 & 1.38428 & 1.71732 & 1.61502 \\
0.50 & $(0.403163,1,0.35716)$ & 0.277539 & 0.24633 & 0.08087 & 0.817571 & 1.14735 & 1.34860 & 1.68156 & 1.57926 \\
1 & $(0.403300,1,0.35849)$ & 0.113311 & 0.24590 & 0.08065 & 0.816265 & 1.14598 & 1.34719 & 1.68015 & 1.57784 \\
2 & $(0.403379,1,0.35922)$ & 0.018881 & 0.24580 & 0.08059 & 0.815909 & 1.14560 & 1.34680 & 1.67976 & 1.57745 \\
3 & $(0.403405,1,0.35946)$ & 0.003146 & 0.24577 & 0.08057 & 0.815792 & 1.14547 & 1.34667 & 1.67963 & 1.57732 \\
5 & $(0.403426,1,0.35966)$ & 0.000087 & 0.24575 & 0.08055 & 0.815695 & 1.14537 & 1.34656 & 1.67952 & 1.57104 \\
\hline
\end{tabular}

Source: Own elaboration

In this second optimization, the results in Table 2 show that the optimal point slightly varies in spite of the increase in the initial level of reserves. However, as expected, the minimal ruin probability decreases when the initial reserves increase. At the optimal point, the behaviour of the expectation, the variance, the $V a R$ and the $T V a R$ is not monotone with respect to the initial reserves. All these risk measures slightly increase, from $u=0$, and then slowly decrease as the initial reserves are increased.

With the threshold proportional reinsurance, the insurer can always obtain a lower ruin probability than with the proportional one (with a constant retention level). In Table 3, the differences of these two minimum probabilities of ruin (the first one attained with proportional reinsurance and the second one attained with threshold proportional reinsurance) are shown, in relative values, for different $u$. These relative differences are less important when the initial reserves are small and that these differences increase with respect to the initial reserves, up to a specific bound (in this case a $15 \%$ approximately).

Optimization and decision problems including the deficit at ruin if ruin occurs. Does an optimal reinsurance strategy such that minimizes the different risk measures of the deficit at ruin if ruin occurs exist? The answer to this problem is no, because the optimal strategy would be to retain nothing.

Then, let us consider the deficit at ruin if ruin occurs as an additional criterion to the ruin probability. We have seen (Tables 1 and 2) that for a fixed $u$, the minimum ruin probability that can be attained with a threshold proportional strategy is always lower than the corresponding one with proportional reinsurance. 
Tabla 3. Relative values with respect to proportional reinsurance.

\begin{tabular}{rc}
\hline$u$ & $\frac{\psi_{k^{*}}(u)-\psi\left(b^{*}, k_{1}^{*}, k_{2}^{*}\right)}{\psi_{k^{*}}(u)} \times 100$ \\
\hline 0 & 9.6998 \\
0.25 & 13.708 \\
0.5 & 13.739 \\
1 & 14.352 \\
2 & 14.662 \\
3 & 14.766 \\
5 & 14.849 \\
\hline
\end{tabular}

Source: Own elaboration

For a fixed $u$, we can obtain all the equivalent strategies to the optimal one with proportional reinsurance, in the sense that with all these strategies the insurer obtains the same probability of ruin. Then, the risk measures (expectation, Value at Risk and Tail Value at Risk) related to the deficit at ruin if ruin occurs are taken as an additional decision criterion to choose between these strategies.

Lets consider, without loss of generality, that $u=0.25$. The minimum ruin probability is 0.497108 , with $k=0.466294$. We obtain an infinite number of $\left(b, k_{1}, k_{2}\right)$ that also allows obtaining this probability of ruin, with a bounded value for $b, 0 \leq b \leq 2.99566$. Figure 1 includes, for several $b$, the values of $k_{1}$ and $k_{2}$ that give the same ruin probability 0.497108 ; the combination $k_{1}=k_{2}=0.466294$ is a specific point of all these curves (the point where all of them coincide). For the insurer, a proportional reinsurance with a retention level 0.466294 is indifferent to all

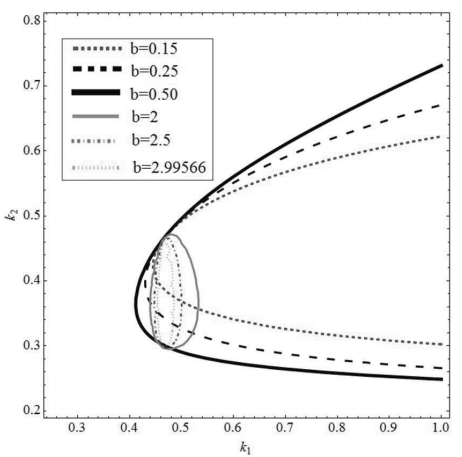

Fig. 1. Level curves of $\psi(u=0.25)=0.497108$ for some levels of $b$ (Source: Own elaboration).

these other threshold proportional reinsurance strategies if the insurer only considers the ruin probability. But what happens as regards the deficit at ruin? Let us focus, for instance, on the case $b=0.5$. In Figure 2, this curve is represented again and the point corresponding to the proportional reinsurance is marked with big black dot while other selected points are marked in gray. In Figure 3, the different risk measures of the deficit at ruin, $E[Y], \operatorname{VaR}_{p}[Y], T V a R_{p}[Y]$, for $p=0.95, p=0.99$ and $p=0.995$, are depicted. If we consider the expected deficit at ruin if ruin occurs, for $b=0.5$ (see Figure 3), we conclude that all the threshold strategies with retention levels $k_{1}<0.466294=k^{*}$ and $k_{2}<0.466294=k^{*}$ are best options than the proportional one, 


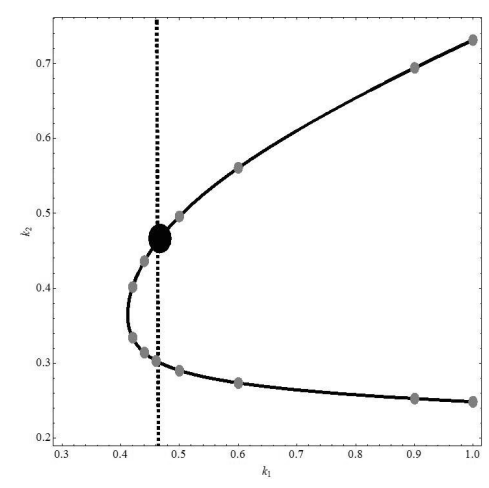

Fig. 2. Level curve of $\psi(u=0.25)=0.497108$ for $b=0.5$ (Source: Own elaboration).
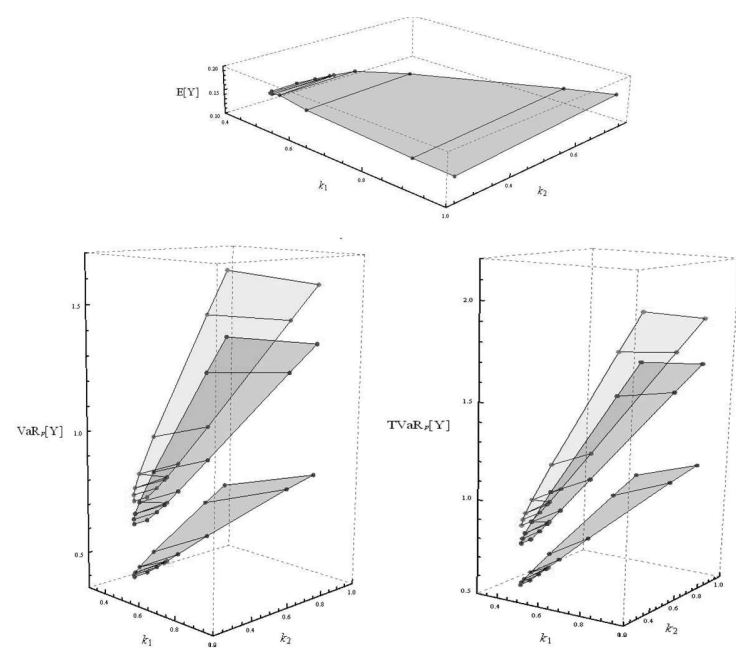

Fig. 3. $E[Y], \operatorname{VaR}_{p}[Y]$ and $T V a R_{p}[Y]$ for $p=0.95$ (down), $p=0.99$ (middle) and $p=0.995$ (up) (Source: Own elaboration).

because the expected deficit at ruin if ruin occurs is lower. This result can be extended to the other measures, $V a R_{p}$ and $T V a R_{p}$, for different levels $p$.

Let us consider also the other possible combination $\left(b, k_{1}, k_{2}\right)$ with $0 \leq b \leq 2.99566$, which are equivalent to the proportional one $\left(k_{1}=k_{2}=k^{*}=0.466294\right)$. In Figure 4 , the expectation of the deficit at ruin if ruin occurs is plotted for some of these combinations with different $b$. We observe that not all of these combinations must fulfill the condition $k_{1} \leq k^{*}$ and $k_{2} \leq k^{*}$, in order to improve the expectation of the deficit at ruin if ruin occurs. A similar conclusion is reached for the other risk measures.

\section{Concluding remarks}

In this paper, we consider the classical risk theory model assuming a Poisson process and an individual claim amount phase-type distributed, modified with a proportional reinsurance with a retention level that is not constant and depends on the level of the surplus. This type of reinsurance, called threshold proportional reinsurance, has been first defined and studied in some of our previous papers [3,4], and includes, as a particular case, the classical proportional reinsurance with constant 


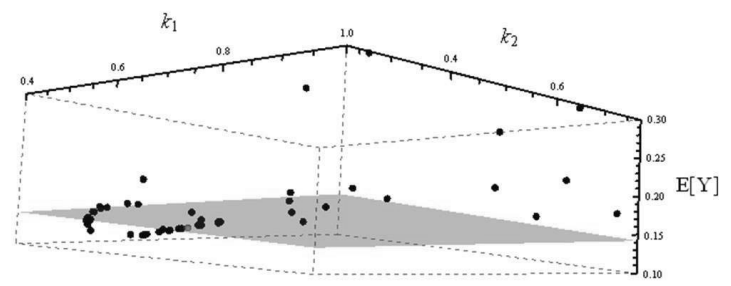

Fig. 4. $E[Y]$ for $u=0.25$ and different $b$ between $0 \leq b \leq 2.99566$ in a threshold proportional reinsurance (Source: Own elaboration).

retention level.

The main contribution of this paper consists on the study of the effect of the threshold proportional reinsurance on the probability of ruin and on the other risk measures related with the deficit at ruin. The Gerber-Shiu function is used as the mathematical tool in order to obtain general results that can be translated into explicit expressions for phase-type 2 distribution. We also perform a comparative analysis with the proportional reinsurance.

Regarding the probability of ruin of the insurer, it can be minimized choosing an appropriate constant retention level or, in an alternative way, using an appropriate combination of two different retention levels and a threshold surplus level, $b$, to change from one retention level to the other. From our analysis, we conclude that the threshold proportional reinsurance is the best option for the insurer if he takes his decisions looking only at the ruin probability, because the threshold proportional reinsurance allows him reducing the ruin probability without increasing the initial capital. This superiority of the threshold proportional reinsurance is stressed (reinforced) when the insurer considers also the random variable deficit at ruin if ruin occurs to take his decisions. We have seen in our examples that, with the threshold proportional reinsurance, the insurer can improve (reduce) the expectation (and the $V a R$ and the $T V a R$ ) of the deficit at ruin if ruin occurs with the same ruin probability than the best proportional reinsurance.

\section{Acknowledgments}

We thank the referees for useful remarks and suggestions. This research was supported by Spanish Ministerio de Educación y Ciencia (ECO2010-22065-C03-03) and the funded chair ICEA-UB on Insurance and Pensions Funds.

\section{References}

1. A. Balbás, B. Balbás and A. Heras, "Stable solutions for optimal reinsurance problems involving risk measures", Eur. J. Oper. Res. 214 (2011) 796-804.

2. A. Balbás, B. Balbás and R. Balbás, "Optimal reinsurance: A risk sharing approach", Risks 1 (2013) 45-56.

3. A. Castañer, M. M. Claramunt and M. Mármol, "Deficit at ruin with threshold proportional reinsurance", Insur. Mark. Co. Anal. Actuar. Comput. 1 (2010) 38-44.

4. A. Castañer, M. M. Claramunt and M. Mármol, "Ruin probability and time of ruin with a proportional reinsurance threshold strategy", TOP 20 (2012) 614-638.

5. M. L. Centeno, "Measuring the effects of reinsurance by the adjustment coefficient", Insur. Math. Econ. 5 (1986) 169-182.

6. M. L. Centeno, "Measuring the effects of reinsurance by the adjustment coefficient in the Sparre Anderson model", Insur. Math. Econ. 30 (2002) 37-49.

7. M. L. Centeno, "Dependent risks and excess of loss reinsurance", Insur. Math. Econ. 37 (2005) 229-238. 
8. L. Gajek and D. Zagrodny, "Optimal reinsurance under general risk measures", Insur. Math. Econ. 34 (2004) 227-240.

9. M. L. Centeno and O. Simões, "Optimal reinsurance", Rev. Real Acad. Cienc. Exactas Fis. Nat. Ser. A-Mat. 103 (2009) 387-404.

10. H. U. Gerber and E. S.W. Shiu, "On the time value of ruin", N. Am. Actuar. J. 2 (1998) 48-72.

11. S. Li and J. Garrido, "On a class of renewal risk models with a constant dividend barrier", Insur. Math. Econ. 35 (2004) 691-701.

12. A. Balbás, B. Balbás and A. Heras, "Optimal reinsurance with general risk measures", Insur. Math. Econ. 44 (2009) 374-384.

13. A. Castañer, M. M. Claramunt and C. Lefèvre, "Survival probabilities in bivariate risk models, with application to reinsurance", Insur. Math. Econ. 53 (2013) 632-642.

14. D. S. Dimitrova and V. K. Kaishev, "Optimal joint survival reinsurance: An efficient frontier approach", Insur. Math. Econ. 47 (2010) 27-35.

15. V. K. Kaishev and D. S. Dimitrova, "Excess of loss reinsurance under joint survival optimality", Insur. Math. Econ. 39 (2006) 376-389.

16. S. Asmussen, Applied Probability and Queues (Springer, New York, 2003).

17. G. Latouche and V. Ramaswami, Introduction to Matrix Analytic Methods in Stochastic Modeling (Society for Industrial and Applied Mathematics, Philadelphia, 1999).

18. M. F. Neuts, Matrix-Geometric Solutions in Stochastic Models: An Algorithmic Approach (The Johns Hopkins University Press, Baltimore, 1981).

19. M. F. Neuts, Structured Stochastic Matrices of $M / G / 1$ Type and Their Applications (Marcel Dekker Inc, New York, 1989).

20. C. A. O'Cinneide, "Characterization of phase-type distributions", Commun. Stat. Stoch. Models 6 (1990) $1-57$.

21. S. Asmussen and H. Albrecher, Ruin Probabilities (World Scientific, Singapore, 2010).

22. S. Asmussen and T. Rolski, "Computational methods in risk theory: A matrix-algorithmic approach", Insur. Math. Econ. 10 (1992) 259-274.

23. S. Asmussen and M. Bladt, "Renewal theory and queueing algorithms for matrix-exponential distributions", in Matrix-Analytic Methods in Stochastic Models, eds. A. S. Alfa and S. R. Chakravarthy (Marcel Dekker Inc, New York, 1996) pp. 313-341.

24. A. I. Bergel and A. D. Egídio dos Reis, "Further developments in the erlang(n) risk process", Scand. Actuar. J. 1 (2015) 32-48.

25. M. Bladt, "A review on phase-type distributions and their use in risk theory", Astin Bull. 35 (2005) 145-161.

26. E. V. Rodríguez-Martínez, R. M. R. Cardoso and A. D. Egídio dos Reis, "Some advances on the erlang(n) dual risk model", Astin Bull. 45 (2015) 127-150.

27. T. Rolski, H. Schmidli, V. Schmidt and J. Teugels, Stochastic Processes for Insurance and Finance (Wiley Series in Probability and Statistics, Chichester, 1999).

28. D. C. M. Dickson and C. Hipp, "Ruin problems for phase-type(2) risk processes", Scand. Actuar. J. 2 (2000) 147-167.

29. S. Drekic, D. C. M. Dickson, D. A. Stanford and G. E. Willmot, "On the distribution of the deficit at ruin when claims are phase-type", Scand. Actuar. J. 2 (2004) 105-120.

30. A. Castañer, M. M. Claramunt and M. Mármol, "Influencia del reaseguro proporcional en las medidas de solvencia del asegurador", in Investigaciones en Seguros y Gestión de Riesgos: RIESGO 2007, eds J. M. Sarabia and M. Guillén (Ediciones TGD, Santander, 2007), pp. 87-101.

31. C. Dutang, V. Goulet and M. Pigeon, "actuar: An R package for actuarial science", J. Stat. Softw. 25 (2008) $1-37$.

32. J. Cai and H. Li, "Conditional tail expectations for multivariate phase-type distributions", J. Appl. Probab. 42 (2005) 810-825.

33. H. U. Gerber, M. J. Goovaerts and R. Kaas, "On the probability and severity of ruin", Astin Bull. 17 (1987) 151-163.

34. C. Hipp, "Speedy convolution algorithms and panjer recursions for phase-type distributions", Insur. Math. Econ. 38 (2006) 176-188. 


\section{Appendix A. Proof of Proposition 1}

Consider that $f(x)$ is the density function of a phase-type distribution satisfying (7), i.e., a differential equation of order $N$

$$
\sum_{i=0}^{N} b_{i} f^{(i)}(x)=0
$$

with $b_{0}=1, b_{i}, i \geq 1, \ldots, N \in R$ and $f^{(0)}(x)=f(x)$ [34].

From (A.1), it is straightforward to obtain

$$
f^{(N)}(x)=-\frac{1}{b_{N}} \sum_{i=0}^{N-1} b_{i} f^{(i)}(x) .
$$

For $0<u<b$, we need some previous results:

Let us define $I N_{h}$ as the $h$-th integral,

$$
I N_{h}=\int_{0}^{\frac{u}{k_{1}}} \phi_{1}\left(u-k_{1} x\right) f^{(h)}(x) d x,
$$

being $h=0, \ldots, N$ and $f^{(0)}(x)=f(x)$. We need the following properties of $I N_{h}$ :

i) The derivative of $I N_{h}$ with respect to $u$ is

$$
I N_{h}^{\prime}=\frac{f^{(h)}(0)}{k_{1}} \phi_{1}(u)+\frac{I N_{h+1}}{k_{1}},
$$

ii) The $h$-th derivative of $I N_{0}$ with respect to $u$ is

$$
I N_{0}^{(h)}=\frac{I N_{h}}{k_{1}^{h}}+\sum_{s=0}^{h-1} \frac{\phi_{1}^{(s)}(u)}{k_{1}^{h-s}} f^{(h-1-s)}(0),
$$

where $1 \leq h \leq N$.

iii) From (A.2) we can obtain $I N_{N}$,

$$
I N_{N}=-\frac{1}{b_{N}} \sum_{h=0}^{N-1} b_{h} I N_{h} .
$$

For $w(l, j)=w(j)$, we define now $I \xi_{h}$ as the $h$-th integral

$$
I \xi_{h}=\int_{\frac{u}{k_{1}}}^{\infty} w\left(k_{1} x-u\right) f^{(h)}(x) d x
$$

being $h=0, \ldots, N$ and $f^{(0)}(x)=f(x)$. Some useful properties of $I \xi_{h}$ are,

i) The derivative of $I \xi_{h}$ with respect to $u$ is

$$
I \xi_{h}^{\prime}=\frac{1}{k_{1}} I \xi_{h+1} .
$$

ii) The $h$-th derivative of $I \xi_{0}$ with respect to $u$ is

$$
I \xi_{0}^{(h)}=\frac{1}{k_{1}^{h}} I \xi_{h}
$$

where $1 \leq h \leq N$.

iii) From (A.2) we can obtain $I \xi_{N}$

$$
I \xi_{N}=-\frac{1}{b_{N}} \sum_{h=0}^{N-1} b_{h} I \xi_{h} .
$$


The integro-differential equation (4) and its derivatives with respect to $u$ until $N+1$ using (A.4) are

$$
\begin{aligned}
\phi_{1}^{\prime}(u)= & \frac{\lambda+\delta}{c_{1}} \phi_{1}(u)-\frac{\lambda}{c_{1}} I N_{0}-\frac{\lambda}{c_{1}} I \xi_{0}, \\
\phi_{1}^{(h+1)}(u)= & \frac{\lambda+\delta}{c_{1}} \phi_{1}^{(h)}(u)-\frac{\lambda}{c_{1}} I \xi_{0}^{(h)} \\
& -\frac{\lambda}{c_{1}}\left(\frac{I N_{h}}{k_{1}^{h}}+\sum_{s=0}^{h-1} \frac{\phi_{1}^{(s)}(u)}{k_{1}^{h-s}} f^{(h-1-s)}(0)\right), 1 \leq h \leq N .
\end{aligned}
$$

And isolating $I N_{0}$ and $I N_{h}$ in (A.10) and (A.11) and substituting in (A.5), and rearranging terms,

$$
\begin{aligned}
I N_{N} & =\frac{1}{b_{N}} I \xi_{0}+\frac{1}{b_{N}} \sum_{h=1}^{N-1} b_{h} k_{1}^{h} I \xi_{0}^{(h)}+\sum_{s=0}^{N} \phi_{1}^{(s)}(u) D_{s} \\
& =\frac{1}{b_{N}}\left(\sum_{h=0}^{N-1} b_{h} k_{1}^{h} I \xi_{0}^{(h)}\right)+\sum_{s=0}^{N} \phi_{1}^{(s)}(u) D_{s}
\end{aligned}
$$

with

$$
D_{s}= \begin{cases}\frac{1}{b_{N}} \sum_{h=1}^{N-1} b_{h} f^{(h-1)}(0)-\frac{\lambda+\delta}{b_{N} \lambda}, & s=0 \\ \frac{c_{1} b_{s-1} k_{1}^{s-1}}{b_{N} \lambda}-\frac{(\lambda+\delta) b_{s} k_{1}^{s}}{b_{N} \lambda}+\frac{k_{1}^{s}}{b_{N}} \sum_{h=s+1}^{N-1} b_{h} f^{(h-1-s)}(0), & s=1, \ldots, N-1 \\ \frac{c_{1} b_{N-1} k_{1}^{N-1}}{b_{N} \lambda}, & s=N .\end{cases}
$$

Finally, substituting (A.12) in (A.11), (10) is obtained. For $u>b$, we can obtain $\phi_{2}^{(N+1)}(u)$ by an analogous process substituting $c_{1}, k_{1}$ and $\phi_{1}(u)$ by $c_{2}, k_{2}$ and $\phi_{2}(u)$. 